\title{
Comparison between front- and back-gating of Silicon Nanoribbons in real-time sensing experiments
}

\author{
E. Accastelli ${ }^{1}$, G. Cappi ${ }^{1}$, J. Buckley ${ }^{2}$, Thomas Ernst ${ }^{2}$ and C. Guiducci ${ }^{1{ }^{1 *}}$
}

\begin{abstract}
Field-effect transistors (FETs) with open gate structures such as Silicon Nanoribbons (SiNRs) are promising candidates to become general platforms for ultrasensitive, labelfree and real-time detection of biochemical interactions on surface. This work proposes and demonstrates the viability of a solution for integrating $\mathrm{Ag} / \mathrm{AgCl}$ reference electrodes with the microfluidics. A comparison between different polarization schemes is carried out with an analysis of the respective advantages and disadvantages.
\end{abstract}

Index Terms - silicon nanoribbons, front-gating, back-gating, microfluidics, reference electrode.

\section{INTRODUCTION}

Field-effect transistors (FETs) with open gate structures such as Silicon Nanoribbons (SiNRs) are promising candidates to become general platforms for ultrasensitive label-free and real-time detection of biochemical interactions on surface [13].

A SiNR molecular sensor operates as a highly sensitive nanoscale FET in which the gate terminal is removed so that the charge distribution in the nearby of the exposed channel directly modulates its surface potential, converting a chemical signal into an electrical one.

The nanometric thickness of the silicon device causes its conductance to be significantly affected by the surface charged species at the SiNR/electrolyte interface. The impact of the surface charges on the silicon material extends over the Thomas-Fermi screening length, which is set by the technological parameters such as the substrate doping [4]. The channel thickness must be smaller than this screening length in order to achieve high sensitivity to surface charge.

On the other side, the surface charge sensitivity is not limited to the charges on the surface of the device but extend toward the Debye screening length in the liquid, determined by the ionic force of the solution.

During the last decades, the miniaturization of FET-based sensors has been the object of extensive research.

On one hand, this field of research has been fostered by the advancements in silicon micromachining technology, which guarantees precise control of dimensions, reproducibility,

1 Ecole Polytechnique Fédérale de Lausanne (EPFL), Lausanne, Switzerland.

2 Commissariat à l'énergie atomique et aux énergies alternatives (CEA-LETI), MINATEC, Grenoble, France

*Email: carlotta.guiducci@epfl.ch excellent uniformity and possibility of cost-efficient mass production [5].

On the other hand, the development of microfluidics has made possible easy, fast and well-controlled biochemical reactions on the sensing areas.

The advantages of this approach include reduced solvents and reagents consumption, shorter diffusion times, portability and ease of integration with other miniaturized devices.

Nevertheless, the fabrication and integration of a reliable miniaturized reference electrode (RE), which is required in most electrochemical measurements, still represents a challenge [6].

As a consequence, many sensing experiments in wet environment are still conducted in macroscopic chambers, either open or closed, filled with a solution containing the analytes of interest. Although this setup allows the use of commercial type RE, the lack of a microfluidic apparatus prevents the possibility of real-time kinetics measurements.

\section{SENSING PLATFORM}

\section{A. Technology}

The SiNRs are fabricated from Silicon-on-Insulator (SOI) wafers. The wafers feature a $200 \mathrm{~nm}$ top silicon layer on a $400 \mathrm{~nm}$ thick $\mathrm{SiO}_{2}$ insulating bulk oxide (BOX).

The silicon top layer is first thinned down by several steps of silicon thermal oxidation/desoxidation to obtain a $50 \mathrm{~nm}$ silicon top layer, corresponding to the height of the SiNRs. In order to obtain n-type devices, the wafers are doped by ion implantation with boron for the SiNRs $\left(1 \times 10^{16} \mathrm{~cm}^{-3}\right)$ body and phosphorous for the source and drain diffusions $\left(5 \times 10^{18} \mathrm{~cm}^{-3}\right)$, respectively.

The SiNRs are patterned using deep ultraviolet (DUV) or e-beam lithography and etched by reactive ion etching (RIE) (Fig. 1). The SiNRs body is covered by a to high quality $3 \mathrm{~nm} \mathrm{SiO}_{2}$ gate oxide is grown by thermal dry oxidation while the rest of the chip is passivated by a multilayered insulator.

The SiNRs can be operated by two different polarization schemes: back-gating and front-gating. The back-gating solution consists in controlling the device through the bulk (back-gate contact) $\mathrm{V}_{\mathrm{BG}-\mathrm{S}}$ (threshold voltage in the $9-11 \mathrm{~V}$ range). In the case of front-gating instead, a voltage $\mathrm{V}_{\mathrm{FG}-\mathrm{S}}$ between the electrolyte solution contacted by a RE 

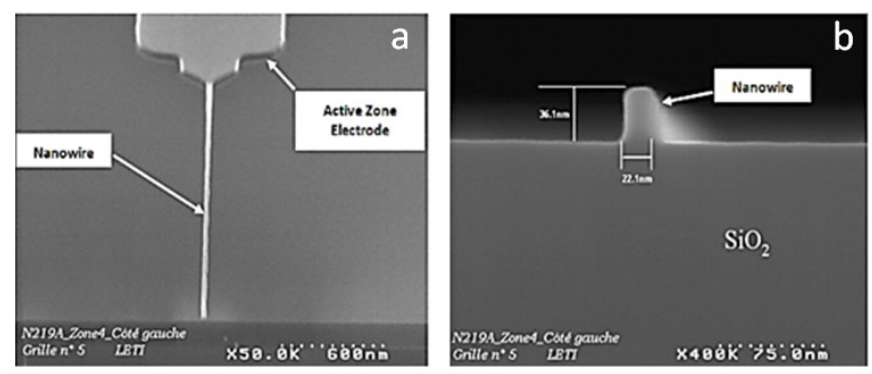

Fig. 1. SEM images of a SiNR: (a) Top view, (b) Cross-section. When the width of the SiNR is comparable with the thickness, as in this case, we can call the structure a nanowire (NW). SEM pictures courtesy of CEA-LETI (Grenoble, France).

(front-gate) and the source diffusion is employed. The threshold voltage range is $4-6 \mathrm{~V}$ in case of back-gating and $1.4-1.5 \mathrm{~V}$ in case of front-gating.

The dimensions of the devices characterized in this work go from $0.11 \mu \mathrm{m}$ to $1 \mu \mathrm{m}$ in width and from $0.5 \mu \mathrm{m}$ to $8 \mu \mathrm{m}$ in length.

The fabrication process was entirely developed at CEA-LETI (Grenoble, FR). A detailed description of the top-down fabrication process can be found in [7].

\section{B. Microfluidics}

Among the many different types of REs, $\mathrm{Ag} / \mathrm{AgCl}$ electrodes have been the object of extensive research and development, especially in the frame of electrochemical applications $[5,8]$. $\mathrm{Ag} / \mathrm{AgCl}$ pseudo-RE can be obtained starting from pure silver by chemical anodization in chlorine ions solutions (e.g., $\mathrm{KCl}$ ) or by plasma treatments in chlorine chambers [8]. In our setup, silver L-shaped tubes are anodized in $0.1 \mathrm{M}$ $\mathrm{KCl}$ solution, in order to obtain a $5 \mu \mathrm{m}$ thick $\mathrm{AgCl}$ metal salt layer.

These tubes are inserted into a microfluidic chip, serving both as REs and as inlet/outlet of the system.

The microchannels are realized with a chemical resistant double-coated tape (3M 9086), patterned by laser micromachining. The height of the channels is defined by the thickness of the tape $(190 \mu \mathrm{m})$. A Poly(methyl methacrylate) (PMMA) cap is placed on top to seal the channels and the inlets and outlets tubes inserted. A sealing polymer is used to avoid fluid leakages from the inlets/outlets (Fig. 2).

During measurements, the solutions are fluxed by means of an external syringe pump (Harvard Apparatus).

The described microfluidic setup is a compact and effective solution that integrates $\mathrm{Ag} / \mathrm{AgCl} \mathrm{REs}$, microfluidics and sensing nanodevices.

\section{Electrical setup}

The electrical measurements were performed by means of a semiconductor parameter analyzer (Agilent 4156C) used both in sweep and sampling mode. We polarized the SiNRs in saturation with constant gate and drain voltages.

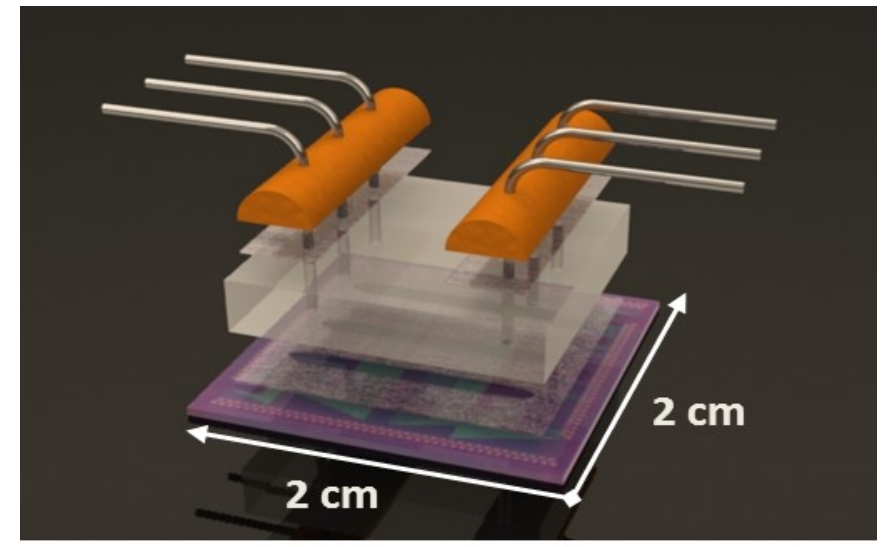

Fig. 2. Exploded view of the microfluidic setup. From bottom to top we can see the NR chip, the double-coated tape defining three microchannels, the PMMA cap, the sealing polymer and the $\mathrm{Ag} / \mathrm{AgCl}$ inlet and outlets tubes.

\section{RESULTS}

According to the site binding model applied to ISFET devices [9-11], the surface of the gate oxide becomes negatively charged when in contact with electrolyte solutions characterized by $\mathrm{pH}$ values bigger than its isoelectric point ( $\mathrm{pI} \approx 2-3$ in the case of $\mathrm{SiO}_{2}$ ). This phenomenon is due to the deprotonation of the silanol groups. A charged silica surface attracts a layer of counter-ions to maintain the overall charge neutrality, forming an Electrical Double Layer (EDL).

The $\mathrm{pH}$-induced surface potential change $\Delta \Psi_{0}$ is given by:

$$
\Delta \Psi_{0}=-2.3 \cdot(K T / e) \cdot \alpha \cdot \Delta p H
$$

where $K$ is the Boltzmann constant, $T$ is the absolute temperature, $e$ the elementary charge, $\alpha$ is the dimensionless sensitivity parameter given by $\alpha=\left(C_{D L} / C_{B}+1\right)^{-1}$ with $C_{B}$ indicating the surface buffer capacitance determined by the density of active $\mathrm{OH}^{-}$groups on the gate oxide and $C_{D L}$ the double layer capacitance with $0 \leq \alpha \leq 1$.

The ISFET sensitivity approaches the theoretical Nernst limit with $\alpha \approx 1$, i.e. when $C_{B}$ is much larger than $C_{D L}$.

Different $\mathrm{pH}$ solutions in contact with the gate oxide lead to different surface potentials and, as a consequence, difference conductance values at a fixed polarization.

Fig. 3 shows the response of two different SiNRs to $\mathrm{pH}$ variation. The two devices were operated in parallel, first in front-gating and then in back-gating configuration. The drain currents $I_{D S}$ were measured in real-time while the solutions with different $\mathrm{pH}$ were injected into the microfluidics.

As expected, $\mathrm{I}_{\mathrm{DS}}$ of an n-type SiNR decreases when the $\mathrm{pH}$ increases since a higher number of negative charges accumulate on the gate oxide surface. Fig. 3 shows an enhanced sensitivity to $\mathrm{pH}$ change when the two devices are operated in back-gating configuration. When front-gating is used, since both the polarization and interaction of the negative charges with the SiNR take place at the front-gate 


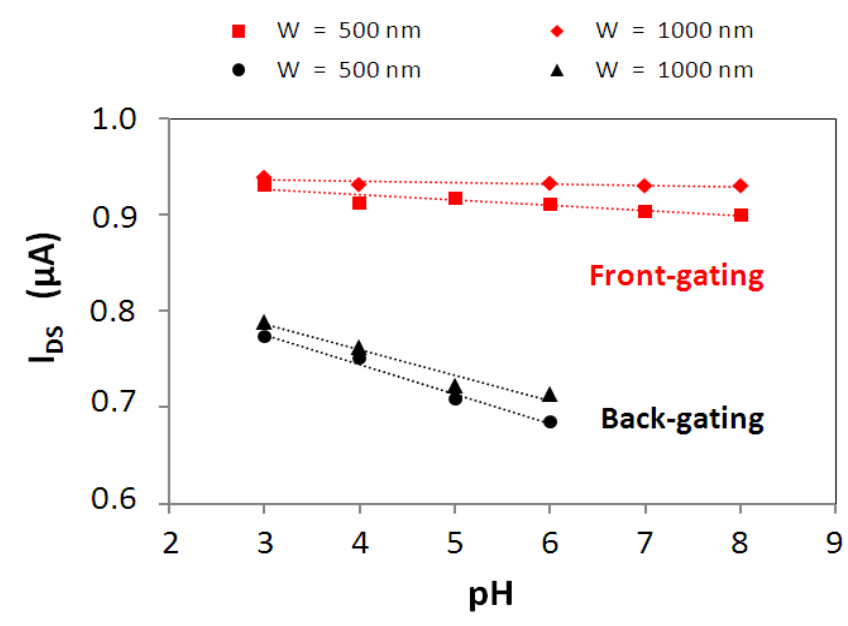

Fig. 3. pH response of the same two SiNRs, polarized through the front-gate (top) and the back-gate (bottom). The lengths of the devices equal the widths.
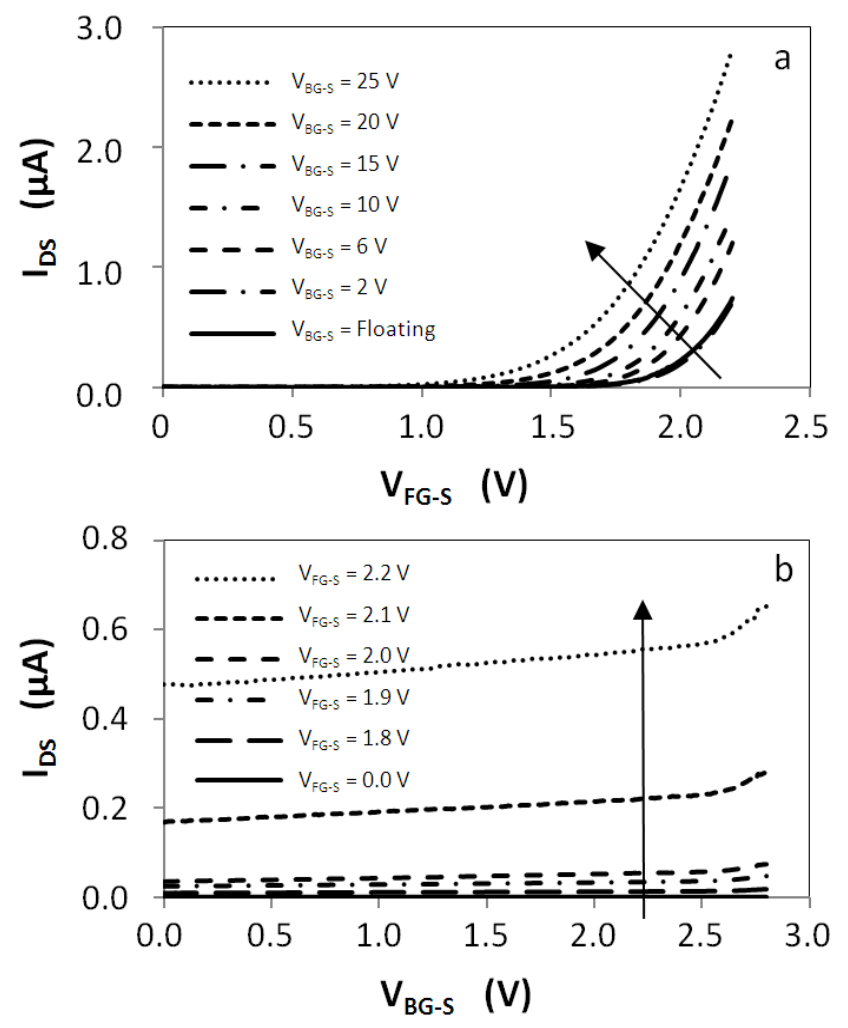

Fig. 4. (a) $I_{D}-V_{F G-S}$ graph plotted for different constant $V_{B G-S}$. The $V_{B G-S}$ only causes a shift in the SiNR threshold voltage. (b) $\mathrm{I}_{\mathrm{D}}-\mathrm{V}_{\mathrm{BG}-\mathrm{S}}$ graph plotted

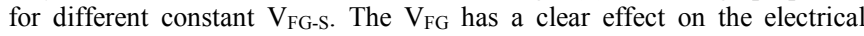
conductance of the device.

side, the change in surface potential leads to an equal shift in the threshold voltage of the device $\left(\Delta \mathrm{V}_{\mathrm{th}, \mathrm{FG}}=\Delta \Psi_{0}\right)$. When back-gating, instead, $\Delta \Psi_{0}$ should be related to the back-gate, through which we actively operate the device. As the
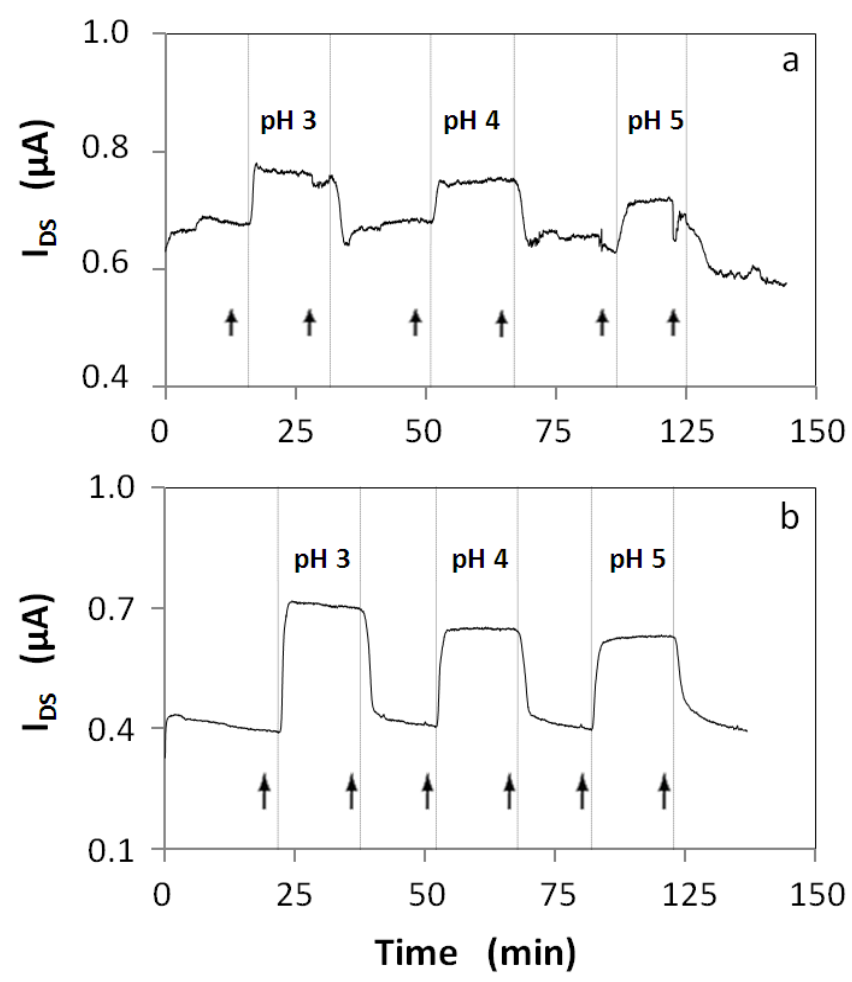

Fig. 5. Real-time drain current measurement in the case of back-gating (a) and front-gating (b) configuration. The arrows and dotted vertical lines indicate the injection of the solution and the actual moment when the solution reaches the sensing area, respectively. The different $\mathrm{pH}$ solutions were prepared with $10 \mathrm{mM}$ phosphate buffers containing $100 \mathrm{mM} \mathrm{KCl}$, which was added to stabilize the $\mathrm{Ag} / \mathrm{AgCl} \mathrm{RE}$. The phosphate solutions were separated by injections of $10 \mu \mathrm{M} \mathrm{KCl}$. The flow rate was $5 \mu 1 / \mathrm{min}$ throughout the whole duration of the experiment.

back-gate capacitance is lower than the front-gate one - due to the thick BOX with respect to the high-quality thin front-gate oxide, the change in surface potential on the front-gate side translates effectively into a bigger back-gate threshold voltage shift $\left(\Delta \mathrm{V}_{\mathrm{th}, \mathrm{BG}}>\Delta \mathrm{V}_{\mathrm{th}, \mathrm{FG}}\right)$. In a setup that polarizes the device with a constant $\mathrm{I}_{\mathrm{DS}}$ and monitors the fluctuations of $\mathrm{V}_{\mathrm{BG}-\mathrm{S}}, \Delta \mathrm{V}_{\mathrm{th}, \mathrm{BG}}$ corresponds to the back-gate voltage shift that compensates for the negative charges accumulated on the front-gate oxide surface. In this kind of configuration ( $\mathrm{I}_{\mathrm{DS}}$ constant), back-gating leads to an enhanced sensitivity in terms of $\Delta \mathrm{V}_{\mathrm{th}} / \Delta \mathrm{pH}$. However, this enhanced response should not be observed when measuring $\mathrm{I}_{\mathrm{DS}}$ at a fixed $\mathrm{V}_{\mathrm{BG}-\mathrm{S}}$, as the $\mathrm{pH}$-induced negative charges should affect the SiNR conductance in the same way. The observed enhanced sensitivity is, at present, the object of further characterization and investigations.

In order to better understand the role played by the possible different polarization schemes on the SiNRs electrical behavior, the gating properties of the front-gate and back-gate on the device have been characterized.

Fig. 4a shows the $I_{D S}-V_{G}$ characteristics of a SiNR $(2375 \mathrm{~nm}$ in length and $500 \mathrm{~nm}$ in width) subjected to a front-gate 


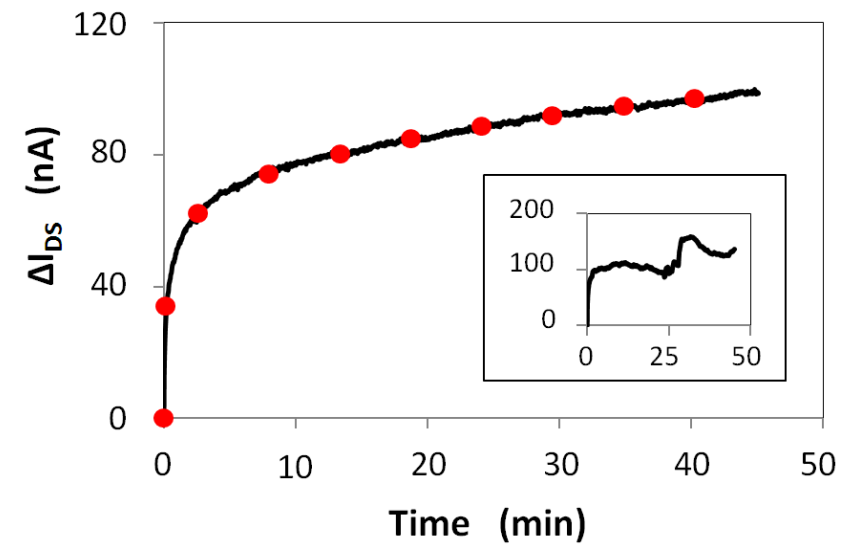

Fig. 6. The drain current drift can be modeled by a stretched-exponential time dependence with a superimposed linear drift. The dots show how well this kind of model can fit the experimental data (line). The fitting parameters in (2) are: $\tau=1000 \mathrm{~s}, \beta=0.22, a=4.5 \mathrm{pA} / \mathrm{s}$ and $b=127 \mathrm{nA}$. (Inset) Change of drain current when the electrolyte is not polarized by means of proper REs.

sweep. Increasing the back-gate voltage leads to a positive shift of the threshold of the device. Nevertheless, the device can neither be switched off $\left(\mathrm{I}_{\mathrm{DS}}=700 \mathrm{nA} @ \mathrm{~V}_{\mathrm{FG}-\mathrm{S}}=2.2 \mathrm{~V}\right.$ and $\left.\mathrm{V}_{\mathrm{BG}-\mathrm{S}}=2 \mathrm{~V}\right)$ nor be switched on $\left(\mathrm{I}_{\mathrm{DS}}=25 \mathrm{pA}\right.$ (a) $\mathrm{V}_{\mathrm{FG}-\mathrm{S}}=0 \mathrm{~V}$ and $\mathrm{V}_{\mathrm{BG}-\mathrm{S}}=20 \mathrm{~V}$ ) through the back-gate.

On the other side, Fig. $4 \mathrm{~b}$ shows that the application of a positive front-gate voltage is sufficient to switch on the device, even if the back-gate is grounded.

It is clear that, given the strong impact of the front-gate on the SiNR behavior, a proper polarization of the electrolyte is crucial to avoid measurement artifacts. In fact, when polarizing the SiNR through the back-gate without any frontgate contact (electrolyte solution left electrically floating) the device current is subjected to noise in particular in correspondence of the change of the buffer solution by injection in the microfluidics (Fig. 5a). On the contrary, the front-gating configuration with back-gate grounded leads to an improved robustness of the measurements towards environmental noise (Fig. 5b).

Moreover, the employment of suitable types of electrodes as $\mathrm{RE}$ is crucial to properly contact the electrolyte solution.

They help reducing, in fact, the drain current noise generated by a floating potential of the solution.

If a proper polarization of the solution is verified in a given setup, the change of the drain current $\Delta I_{D S}$ in time follows an exponential law with an associated linear drift given by the form:

$$
\Delta I_{D}(t)=\left[1-e^{\left(-\frac{t}{\tau}\right)^{\beta}}\right](a \cdot t+b)
$$

where $t$ is the time, $\tau, \beta, a$ and $b$ are fitting parameters describing the stretched-exponential time dependence and the linear time one, respectively. Fig 6 shows the fitting to
(2) of a set of current measurements of a SiNR device ( $\mathrm{W}=0.29 \mu \mathrm{m} ; \mathrm{L}=8 \mu \mathrm{m}$ ) in a $45 \mathrm{~min}$ timeframe. The inset shows the unstable current trend that is obtained by polarizing the solution through non modified silver tubes.

\section{CONCLUSIONS}

In this work, we proposed a compact and effective microfluidic system that solves in a simple and reliable way the problem of integration of a reference electrode within a biochemical sensing platform.

We showed the performance of such implementation in terms of noise reduction when applied to real-time measurements on SiNR-FET sensors.

We also investigated the sensing performance of the SiNRs comparing front- and back-gate polarization schemes, highlighting the advantages and disadvantages of the two approaches. The results of this work clearly show the importance of the polarization scheme in order to achieve high sensitivity, stability and robustness.

\section{ACKNOWLEDGMENT}

This work, scientifically evaluated by the SNSF, has been funded by Nano-Tera.ch (project 128852 "ISyPeM"), an initiative financed by the Swiss Confederation.

\section{REFERENCES}

[1] Y. Cui, et al., "Nanowire Nanosensors for Highly Sensitive and Selective Detection of Biological and Chemical Species," Science, vol. 293, pp. 1289-1292, August 17, 20012001.

[2] M. Curreli, et al., "Real-Time, Label-Free Detection of Biological Entities Using Nanowire-Based FETs," Nanotechnology, IEEE Transactions on, vol. 7, pp. 651-667, 2008.

[3] E. Stern, et al., "Importance of the Debye Screening Length on Nanowire Field Effect Transistor Sensors," Nano Letters, vol. 7, pp. 3405-3409, 2007.

[4] O. Knopfmacher, et al., "Nernst Limit in Dual-Gated Si-Nanowire FET Sensors," Nano Letters, vol. 10, pp. 2268-2274, 2010/06/09 2010.

[5] H. Suzuki, et al., "Microfabricated Liquid Junction $\mathrm{Ag} / \mathrm{AgCl}$ Reference Electrode and Its Application to a One-Chip Potentiometric Sensor," Analytical Chemistry, vol. 71, pp. 5069-5075, 1999.

[6] P. Se-Ik, et al., "Application of a new Cl-plasma-treated $\mathrm{Ag} / \mathrm{AgCl}$ reference electrode to micromachined glucose sensor," Sensors Journal, IEEE, vol. 3, pp. 267-273, 2003.

[7] C. Halté, et al., "Rapid Top-down Fabrication of Si Nanowire and Fully Automated Test Platform: Application to $\mathrm{pH}$," in pHealth, Valencia, 2008.

[8] Polk, et al., $\mathrm{Ag} / \mathrm{AgCl}$ microelectrodes with improved stability for microfluidics vol. 114. Kidlington, ROYAUME-UNI: Elsevier, 2006.

[9] P. Bergveld, "Development of an Ion-Sensitive Solid-State Device for Neurophysiological Measurements," Biomedical Engineering, IEEE Transactions on, vol. BME-17, pp. 70-71, 1970.

[10] P. Bergveld, "Thirty years of ISFETOLOGY: What happened in the past 30 years and what may happen in the next 30 years," Sensors and Actuators B: Chemical, vol. 88, pp. 1-20, 2003.

[11] D. E. Yates, et al., "Site-binding model of the electrical double layer at the oxide/water interface," Journal of the Chemical Society, Faraday Transactions 1: Physical Chemistry in Condensed Phases, vol. 70, pp. 1807-1818, 1974. 